\title{
Pulmonary infection caused by Mycobacterium kansasii: findings on computed tomography of the chest*
}

\author{
Alterações na tomografia computadorizada do tórax em pacientes com infecção pulmonar \\ por Mycobacterium kansasii
}

\author{
Roberto Mogami ${ }^{1}$, Telma Goldenberg ${ }^{2}$, Patricia Gomes Cytrangulo de Marca ${ }^{3}$, Fernanda Carvalho de Queiroz \\ Mello ${ }^{4}$, Agnaldo José Lopes ${ }^{5}$
}

Mogami R, Goldenberg T, Marca PGC, Mello FCQ, Lopes AJ. Pulmonary infection caused by Mycobacterium kansasii: findings on computed tomography of the chest. Radiol Bras. 2016 Jul/Ago;49(4):209-213.

Abstract Objective: To describe the main tomography findings in patients diagnosed with pulmonary infection caused by Mycobacterium kansasii. Materials and Methods: Retrospective study of computed tomography scans of 19 patients with pulmonary infection by $M$. kansasii. Results: Of the 19 patients evaluated, 10 (52.6\%) were male and 9 (47.4\%) were female. The mean age of the patients was 58 years (range, 33-76 years). Computed tomography findings were as follows: architectural distortion, in 17 patients (89.5\%); reticular opacities and bronchiectasis, in 16 (84.2\%); cavities, in 14 (73.7\%); centrilobular nodules, in 13 (68.4\%); small consolidations, in 10 (52.6\%); atelectasis and large consolidations, in 9 (47.4\%); subpleural blebs and emphysema, in 6 (31.6\%); and adenopathy, in 1 (5.3\%).

Conclusion: There was a predominance of cavities, as well as of involvement of the small and large airways. The airway disease was characterized by bronchiectasis and bronchiolitis presenting as centrilobular nodules.

Keywords: Mycobacterium infections, nontuberculous; Tomography, X-ray computed; Lung/pathology.

Resu mo Objetivo: Descrever os achados tomográficos de pacientes com diagnóstico de infecção pulmonar pelo Mycobacterium kansasii.

Materiais e Métodos: Estudo retrospectivo dos exames de tomografia computadorizada do tórax de 19 pacientes com infecção pulmonar pelo M. kansasii.

Resultados: Dos 19 pacientes avaliados, 10 (52,6\%) eram do sexo masculino e 9 (47,4\%) eram do sexo feminino. A média de idade do grupo foi 58 anos, com variação entre 33 e 76 anos. As alterações encontradas nos exames de tomografia computadorizada foram distorção arquitetural em 17 pacientes (89,5\%), opacidades reticulares e bronquiectasias em 16 (84,2\%), cavidades em 14 (73,7\%), nódulos centrolobulares em 13 (68,4\%), pequenas consolidações em 10 (52,6\%), atelectasias e grandes consolidações em 9 (47,4\%), bolhas subpleurais e enfisema em $6(31,6 \%)$ e linfonodomegalias em 1 paciente $(5,3 \%)$.

Conclusão: Houve predomínio de cavidades e do padrão de acometimento de pequenas e grandes vias aéreas. A doença de vias aéreas foi caracterizada por bronquiectasias e bronquiolites que se manifestaram como nódulos centrolobulares.

Unitermos: Infecções por micobactéria não tuberculosa; Tomografia computadorizada; Pulmão.

\section{INTRODUCTION}

Nontuberculous mycobacteria (NTM) are organisms that are ubiquitous in nature and can cause infections in immunocompetent patients. They can be divided into two

* Study conducted at the Centro de Referência Professor Hélio Fraga (CRPHF) of the Escola Nacional de Saúde Pública Sergio Arouca / Fundação Oswaldo Cruz (ENSP) Fiocruz), Rio de Janeiro, RJ, Brazil.

1. PhD, Adjunct Professor of Radiology at the Universidade do Estado do Rio de Janeiro (UERJ), Head of the Department of Radiology at the Hospital Universitário Pedro Ernesto (HUPE), Rio de Janeiro, RJ, Brazil.

2. Master's Student in the Graduate Program in Clinical Medicine at the Universidade Federal do Rio de Janeiro (UFRJ), Physician at the Centro de Referência Professor Hélio Fraga (CRPHF) of the Escola Nacional de Saúde Pública Sergio Arouca / Fundação Oswaldo Cruz (ENSP/Fiocruz), Rio de Janeiro, RJ, Brazil.

3. Graduate Student in Radiology at the Universidade do Estado do Rio de Janeiro (UERJ), Rio de Janeiro, RJ, Brazil.

4. PhD, Director of the Instituto de Doenças do Tórax, Associate Professor of Pulmonology at the Faculdade de Medicina da Universidade Federal do Rio de Janeiro (UFRJ), Rio de Janeiro, RJ, Brazil.

5. PhD, Adjunct Professor of Pulmonology at the Universidade do Estado do Rio de Janeiro (UERJ), Rio de Janeiro, RJ, Brazil. groups, according to the growth pattern: slow and fast. Slowgrowing NTM include Mycobacterium avium, Mycobacterium intracellulare (Mycobacterium avium-intracellulare complex) and Mycobacterium kansasii, whereas fast-growing NTM include Mycobacterium abscessus, Mycobacterium fortuitum and Mycobacterium chelonae ${ }^{(1)}$.

NTM can be isolated in water, soil, milk, and animal flesh. The infection can be acquired by inhalation, ingestion, or direct inoculation. In the case of infection with $M$. kansasii, the main reservoir is in tap water and the contamination is via the airway ${ }^{(2)}$. The disease has a chronic, indolent course, and diagnosis is difficult because isolation of the agent in bronchoalveolar lavage fluid may represent only colonization of the airway ${ }^{(3)}$. The type of NTM most often

Mailing address: Dr. Agnaldo José Lopes. Universidade do Estado do Rio de Janeiro. Rua Araguaia, 1266, Bloco 1/405, Freguesia, Jacarepaguá. Rio de Janeiro, RJ Brazil, 22745-271. E-mail: agnaldolopes.uerj@gmail.com.

Received May 5, 2015. Accepted after revision July 14, 2015. 
isolated is $M$. avium-intracellulare complex ${ }^{(4)}$, followed by M. kansasii( ${ }^{(2,5)}$.

Initially, two patterns of pulmonary involvement by NTM were identified: cavitary disease in the upper lobes; and nodular disease with bronchiectasis, mainly in the middle lobe and lingula ${ }^{(6)}$. The first pattern, which was reported most frequently in the $1980 \mathrm{~s}$, mimicked the classic aspect of tuberculosis. Prior to the advent of computed tomography (CT), authors such as Zvetina et al. ${ }^{(7)}$ wrongly attempted to establish radiographic differences between the cavities caused by $M$. kansasii and those caused by $M$. tuberculosis. The typical patient showing the cavitary disease pattern has some type of comorbidity, such as alcoholism, cancer, chronic obstructive pulmonary disease, cystic fibrosis, bronchiectasis, and pulmonary fibrosis ${ }^{(8)}$. The second pattern occurs predominantly in nonsmoking middle-aged women who have chronic cough and expectoration. In this subtype, conditions that predispose to NTM are uncommon. The disease is known by some as Lady Windermere syndrome ${ }^{(9,10)}$. In a review of the CT scans of 22 patients with NTM, Jeong et al. ${ }^{(11)}$ found the pattern of nodular disease with bronchiectasis to be the most common. The corresponding histopathology finding was bronchiolectasis with bronchial and peribronchial inflammatory infiltrates, with or without granuloma formation. Pulmonary involvement with bronchiectasis and signs of bronchiolitis is not specific to a given strain of mycobacteria ${ }^{(4,11)}$.

In addition to the two patterns mentioned above, Erasmus et al. ${ }^{(3)}$ divided patients infected with NTM into three specific categories, on the basis of clinical and radiological changes: asymptomatic patients with nodules; patients with achalasia and thoracic abnormalities; and immunocompromised patients with thoracic changes. In the last category, the radiological pattern is slightly different ${ }^{(12)}$, the most common findings being interstitial involvement, in 51.3\%; consolidations, in $37.5 \%$; pleural effusion, in $36.3 \%$; and adenopathy, in $31.3 \%$. Matveychuk et al. ${ }^{(5)}$ found differences among the various types of NTM infection in terms of the radiographic findings. The presence of cavities was more common in infections caused by $M$. kansasii, as were lesions in the upper lobes and unilateral lung involvement. Those authors also reported that pleural effusion and mediastinal adenopathy were found in few of the patients infected with M. kansasii ${ }^{(5)}$. Similarly, Shitrit et al. ${ }^{(2)}$ found that, among patients infected with $M$. kansasii, the predominant aspects were cavitary disease (in 54\%) and major involvement of the upper lobes (in 82\%). In both of those studies, the authors observed no pleural effusion or adenopathy in any of the patients ${ }^{(2,5)}$. However, Matveychuk et al. ${ }^{(5)}$ and Shitrit et al. ${ }^{(2)}$ both used routine chest $\mathrm{X}$-ray alone to identify abnormalities in that patient population.

Given that chest CT differs from conventional chest Xray, in terms of sensitivity and specificity, with better spatial resolution and greater discrimination of densities ${ }^{(13,14)}$, it is essential to evaluate its role in the diagnosis of thoracic abnormalities in patients infected with M. kansasii. To our knowledge, there have been no studies aimed at discriminating the CT findings in such patients in Brazil. Because of the difficulty in establishing the diagnosis of NTM lung infections, the aim of this study was to describe the frequency of abnormalities found on CT scans of the chest in a group of patients with pulmonary infection caused by M. kansasii.

\section{MATERIALS AND METHODS}

We evaluated the chest CT scans of 19 patients with proven pulmonary infection caused by $M$. kansasii, treated at our institution between 2006 and 2014. The diagnosis of pulmonary infection with $M$. kansasii (rather than colonization alone) was made by combining clinical, radiological, and microbiological criteria, as recommended by the American Thoracic Society ${ }^{(15)}$. Microbiological confirmation was obtained by applying the following combinations of criteria: three positive cultures with negative smear microscopy results or two positive cultures and one positive sputum smear microscopy; one positive smear microscopy and one positive culture or just one positive culture of bronchoalveolar lavage fluid; one positive culture or one pathology examination showing inflammatory granuloma formation, with or without a smear-positive lung biopsy ${ }^{(16)}$. CT scans, performed in various scanners, produced documentation of sequential slices in a parenchymal window (including highresolution acquisition) and a mediastinal window, without administration of intravenous contrast. The CT scans were interpreted separately and randomly by a thoracic radiologist with more than 10 years of experience in the specialty. The abnormalities were defined and classified in accordance with the criteria established by the Fleischner Society ${ }^{(17)}$ and the Brazilian Illustrated Consensus terminology of descriptors and fundamental patterns on chest CT scans ${ }^{(18)}$. In addition, for the purposes of interpretation, consolidations were divided into large and small, a cut-off value of $3 \mathrm{~cm}$ being adopted to differentiate between the two.

\section{RESULTS}

Among the 19 patients evaluated, the mean age was 58 years (range, 33-76 years), $10(52.6 \%)$ were male, and 9 (47.4\%) were female.

The main changes seen on the 19 CT scans evaluated were architectural distortion, in 17 patients $(89.5 \%)$, as shown in Figure 1; reticular opacities, in 16 (84.2\%), also shown in Figure 1; bronchiectasis, in 16 (84.2\%), as shown in Figures 2 and 3; cavities, in 14 (73.7\%), as shown in Figures 4 and 5; and centrilobular nodules, in $13(68.4 \%)$, as shown in Figures 2 and 3. Other changes were small $(\leq 3 \mathrm{~cm})$ consolidations, in 10 cases $(52.6 \%)$, as shown in Figure 6; atelectasis, in $9(47.4 \%)$, as shown in Figure 1; large (>3 $\mathrm{cm})$ consolidations, also in $9(47.4 \%)$, as shown in Figure 5; subpleural blebs and emphysema, in $6(31.6 \%)$, as shown in Figure 4; and adenopathy, in 1 (5.3\%).

In $10(52.6 \%)$ of the 19 cases, bronchiectasis was observed in the upper lobes, whereas it was observed in the lower 


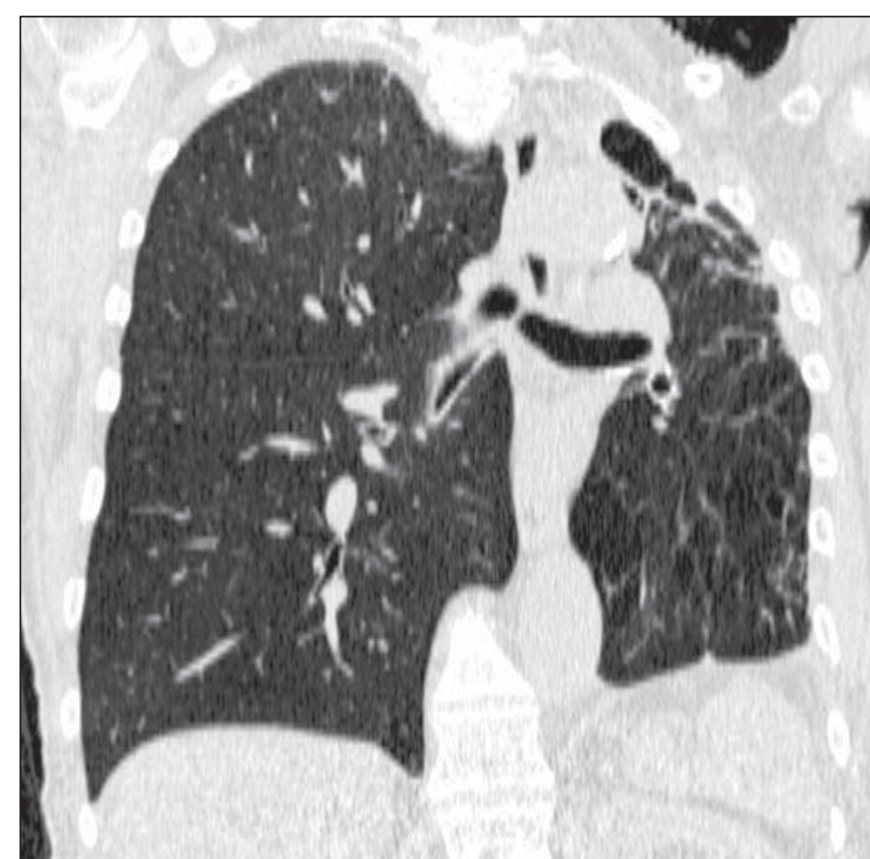

Figure 1. Left lung volume loss, with architectural distortion and reticular opacities.

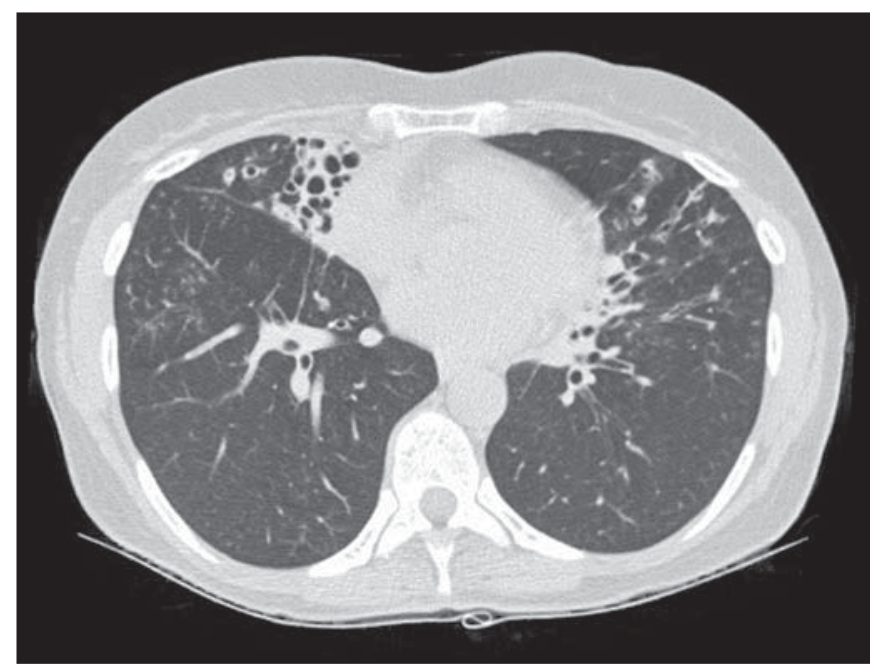

Figure 2. Bronchiectasis and nodules in the middle lobe, lower right lobe, and lingula. In the middle lobe, atelectasis and architectural distortion caused by destruction of the lung parenchyma can be seen.

lobes in 6 cases $(31.6 \%)$ and in the middle lobe/lingula in 3 (15.8\%). One patient $(5.3 \%)$ had calcified nodules and the remaining $18(94.7 \%)$ had centrilobular nodules attributed to filling of the bronchioles (infectious bronchiolitis).

Of the 19 patients, $12(85.7 \%)$ showed cavities in the upper lobes, only two patients (14.3\%) showing cavities in the lower lobes. No cavities were observed in the middle lobe or lingula.

\section{DISCUSSION}

The assessment of pulmonary infections by CT has been the subject of a number of recent publications in the radiology literature of Brazil ${ }^{(19-24)}$. In the present study, the evalu-

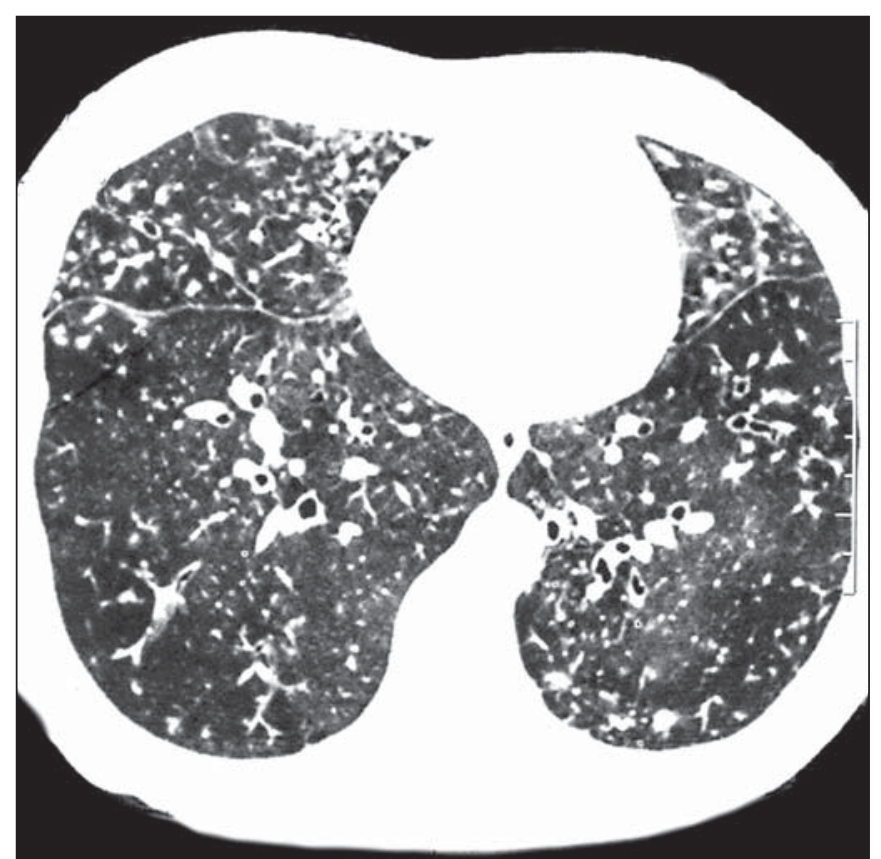

Figure 3. Areas showing a mosaic pattern of attenuation and tree-in-bud opacities.

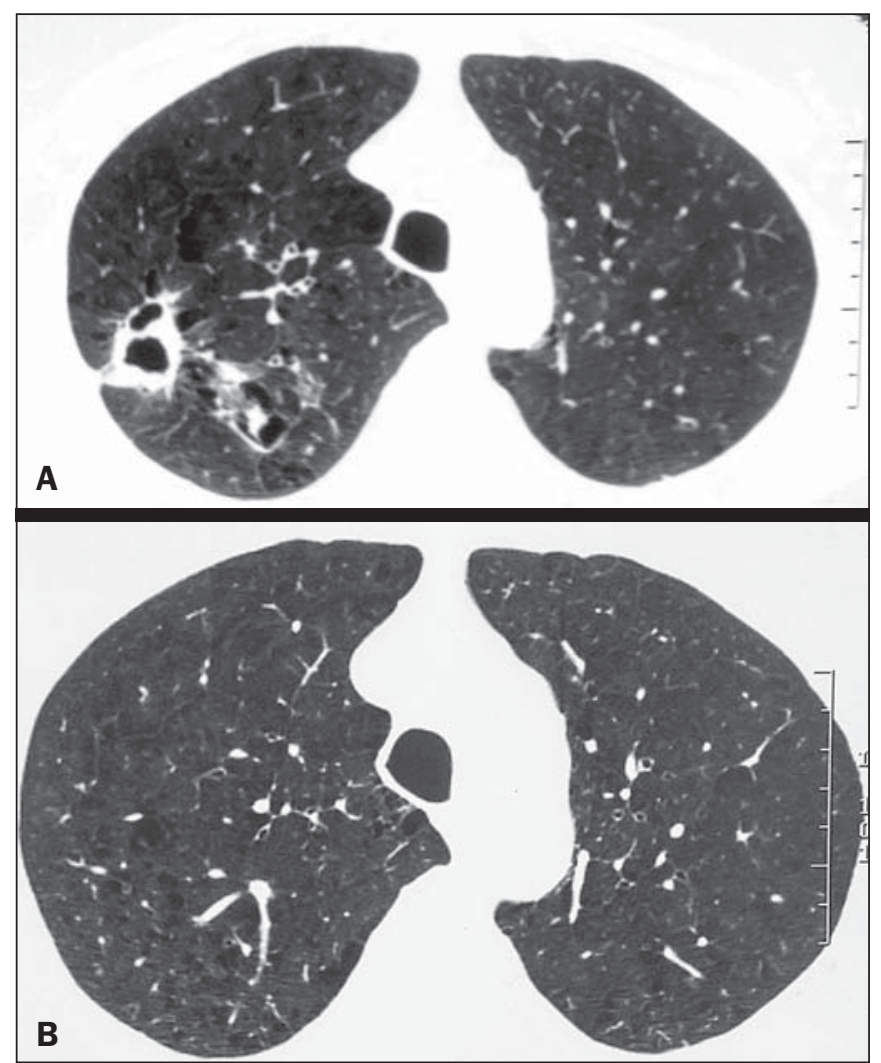

Figure 4. A: Cavity in the right upper lobe, accompanied by signs of centrilobular emphysema. B: After successful treatment, the cavity disappeared.

ation of the changes found on CT scans showed that the main findings, in order of frequency, were architectural distortion, changes in the interstitial space, cavities, and centrilobular nodules. It is known that there are two main patterns of involvement by NTM: cavities in the upper lobes (similar to 


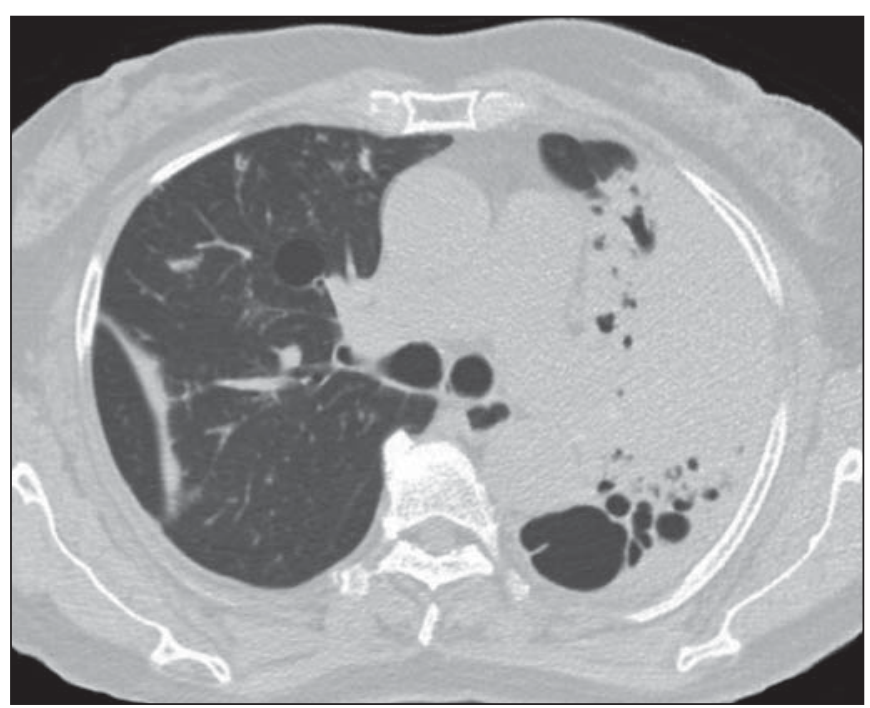

Figure 5. Consolidation and cavity in the left lung.

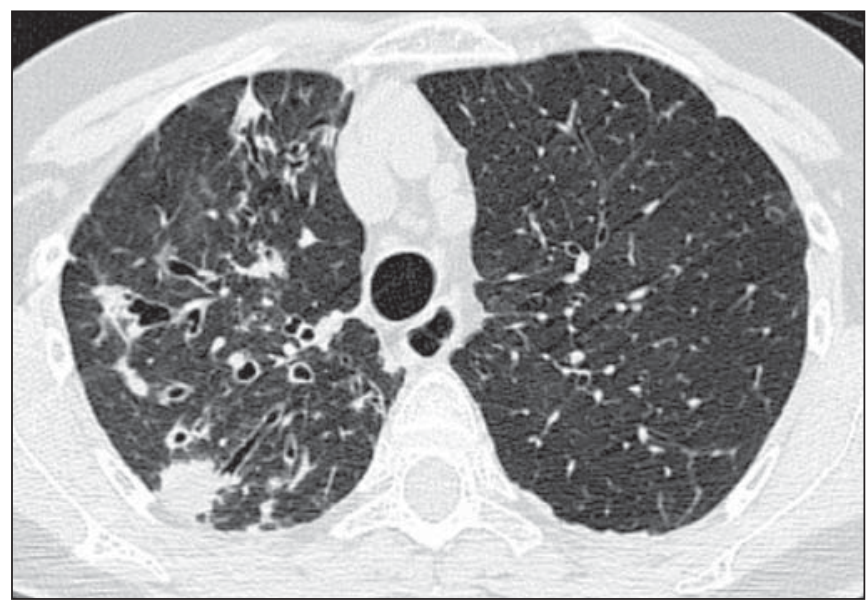

Figure 6. Subsegmental consolidations and bronchiectasis.

tuberculosis) and bronchiectasis/bronchiolitis ${ }^{(6,7,9,10)}$. In our sample, those two patterns of involvement occurred at similar frequencies, different than in the study conducted by Takahashi et al. ${ }^{(25)}$, who, in a sample of 29 patients infected with $M$. kansasii and evaluated by CT, identified cavity lesions in $83 \%$ of cases and bronchiectasis in only $27.6 \%$. It is possible that the differences in the frequencies of comorbidities and rates of tuberculosis prevalence between the populations evaluated explain, at least in part, the discrepancies in the results of the two studies.

Some authors refer to the pattern of preferential involvement of the airways, when bronchiectasis occurs in the middle lobe/lingula and is associated with NTM, as Lady Windermere syndrome ${ }^{(9,10)}$. This syndrome was first described in 1992 by Reich et al. ${ }^{(26)}$, who associated it with the clinical features inspired by the personality of the protagonist of the Oscar Wilde play "Lady Windermere's Fan”, who concealed her cough for social reasons (shame in coughing and expectorating). In Lady Windermere syndrome, in addition to multiple areas of bronchiectasis, there is radiological evidence of centrilobular nodules and a tree-in-bud pattern, together with voluntary cough suppression, leading to a nonspecific inflammatory process, at a site where drainage is difficult, which could explain its pathogenesis ${ }^{(27,28)}$. In our sample, however, bronchiectasis was most prevalent in the upper lobes $(52.6 \%)$. That differs from the distribution reported in the literature for individuals infected with M. kansasii or M. avium-intracellulare complex ${ }^{(5,6)}$.

The high frequency of architectural distortion seen on the CT scans evaluated in the present study might be due to the NTM itself or secondary to other comorbidities that can lead to fibrosis, such as tuberculosis. CT can identify architectural changes that, on conventional X-rays, can be confused with bronchiectasis and areas of fibrosis. In addition, CT clearly demonstrates the important features of the cavities, such as the borders, the wall thickness, and the contents. In the present study, cavitary lesions in the upper lobes predominated, being observed in $85.7 \%$ of the cases, similar to what has been reported by other authors ${ }^{(26)}$.

On the CT scans, we observed large and small consolidations in high proportions of the patients $(47.4 \%$ and $52.6 \%$, respectively). Large consolidations were defined as those $>$ $3 \mathrm{~cm}$ and could represent injuries that converged and expanded to broader regions of the lung parenchyma. In the follow-up of the patients with such consolidations, we observed that, in some cases, the confluence of those consolidations worsened the tomographic alterations ${ }^{(3)}$.

Similar to what was reported in the study conducted by Takahashi et al. ${ }^{(25)}$, we observed a low incidence of adenopathy and pleural lesions. This is important information in Brazil, where the incidence of tuberculosis is very high. That is because a finding of pleural involvement or lymph node enlargement makes a diagnosis of NTM less likely, and when there is concomitant suspicion of tuberculosis, the second condition-association of this two diseases - is more plausible.

Although chronic obstructive pulmonary disease is a predisposing factor for infection with NTM, we observed a low prevalence of emphysematous lesions and pulmonary blebs (31.6\% for both) on CT scans. For the most part, the literature does not mention these findings in radiological examinations of infection with $M$. kansasii ${ }^{(26)}$. In a sample of patients with pulmonary infection caused by M. aviumintracellulare complex, Song et al. ${ }^{(29)}$ also reported a low prevalence of blebs and emphysema (11\% and 32\%, respectively).

A critical analysis of the results of this study and its limitations is relevant. First, because this was a retrospective study based on surveys conducted at different institutions, the documentation did not always refer to images obtained during an expiratory breath hold, which would be important to assess air trapping and consequently (indirectly) bronchiolitis. Second, the CT findings were reviewed by only one radiologist, which makes them susceptible to bias. Third, we did not determine whether the tomographic findings correlated with the clinical findings and with pulmonary 
function. Despite these limitations, we believe our results make an important contribution, given that there have been few studies evaluating tomographic findings in this group of patients. Future studies, using a statistical analysis involving a larger sample, a control group, and longitudinal follow-up of these patients, will confirm our observations.

In conclusion, the predominant features in our sample of 19 patients with pulmonary infection caused by $M$. kansasii were cavities and airway involvement (characterized by bronchiectasis and changes attributed to the filling of the bronchioles).

\section{Acknowledgment}

To Fundação Carlos Chagas Filho de Amparo à Pesquisa do Estado do Rio de Janeiro (Faperj), for the financial support through the Project no. E-26/1 10.255/2014).

\section{REFERENCES}

1. Figueiredo CM. Micobactérias atípicas. In: Silva CIS, D’Ippolito G, Rocha AJ, editores. Tórax. Rio de Janeiro, RJ: Elsevier; 2010. p. 271-6.

2. Shitrit D, Baum GL, Priess R, et al. Pulmonary Mycobacterium kansasii infection in Israel, 1999-2004: clinical features, drug susceptibility, and outcome. Chest. 2006;129:771-6.

3. Erasmus JJ, McAdams HP, Farrell MA, et al. Pulmonary nontuberculous mycobacterial infection: radiologic manifestations. Radiographics. 1999;19:1487-505.

4. Koh WJ, Lee KS, Kwon OJ, et al. Bilateral bronchiectasis and bronchiolitis at thin-section CT: diagnostic implications in nontuberculous mycobacterial pulmonary infection. Radiology. 2005;235: 282-8.

5. Matveychuk A, Fuks L, Priess R, et al. Clinical and radiological features of Mycobacterium kansasii and other NTM infections. Respir Med. 2012;106:1472-7.

6. Rubin SA. Tuberculosis and atypical mycobacterial infections in the 1990s. Radiographics. 1997;17:1051-9.

7. Zvetina JR, Demos TC, Maliwan N, et al. Pulmonary cavitations in Mycobacterium kansasii: distinction from M. tuberculosis. AJR Am J Roentgenol. 1984;143:127-30.

8. Wickremasinghe M, Ozerovitch LJ, Davies G, et al. Non-tuberculous mycobacteria in patients with bronchiectasis. Thorax. 2005; 60:1045-51.

9. Kasthoori JJ, Liam CK, Wastie ML. Lady Windermere syndrome: an inappropriate eponym for an increasingly important condition. Singapore Med J. 2008;49:e47-9.

10. Hartman TE, Jensen E, Tazelaar HD, et al. CT Findings of granulomatous pneumonitis secondary to Mycobacterium avium-intracellulare inhalation: "hot tub lung". AJR Am J Roentgenol. 2007; 188:1050-3.

11. Jeong YJ, Lee KS, Koh WJ, et al. Nontuberculous mycobacterial pulmonary infection in immunocompetent patients: comparison of thin-section CT and histopathologic findings. Radiology. 2004;231: 880-6.

12. dos Santos RP, Scheid KL, Willers DM, et al. Comparative radio- logical features of disseminated disease due to Mycobacterium tuberculosis vs non-tuberculosis mycobacteria among AIDS patients in Brazil. BMC Infect Dis. 2008;8:24.

13. Elicker B, Pereira CA, Webb R, et al. High-resolution computed tomography patterns of diffuse interstitial lung disease with clinical and pathological correlation. J Bras Pneumol. 2008;34:71544.

14. Rodrigues RS, Marchiori E. Tomografia de alta resolução do tórax: padrões básicos. In: Santos AASMD, Nacif MS, editors. Radiologia e diagnóstico por imagem: aparelho respiratório. Rio de Janeiro, RJ: Rubio; 2004. p. 29-50.

15. Griffith DE, Aksamit T, Brown-Elliott BA, et al. An official ATS/ IDSA statement: diagnosis, treatment, and prevention of nontuberculous mycobacterial diseases. Am J Respir Crit Care Med. 2007;1 175:367-416.

16. Haddad DJ, Ide J, Ferrazoli L, et al. Micobacterioses: recomendações para o diagnóstico e tratamento. São Paulo, SP: Secretaria Estadual de Saúde de São Paulo; 2005.

17. Hansell DM, Bankier AA, MacMahon H, et al. Fleischner Society: glossary of terms for thoracic imaging. Radiology. 2008;246:697722.

18. Silva CIS, Marchiori E, Souza Júnior AS, et al. Consenso brasileiro ilustrado sobre a terminologia dos descritores e padrões fundamentais da TC de tórax. J Bras Pneumol. 2010;36:99-123.

19. Zanetti G, Nobre LF, Mançano AD, et al. Pulmonary paracoccidioidomycosis [Which is your diagnosis?]. Radiol Bras. 2014;47(1): xi-xiii.

20. Fernandes MC, Zanetti G, Hochhegger B, et al. Rhodococcus equi pneumonia in an AIDS patient [Which is your diagnosis?]. Radiol Bras. 2014;47(3):xi-xiii.

21. Nishiyama KH, Falcão EAA, Kay FU, et al. Acute tracheobronchitis caused by Aspergillus: case report and imaging findings. Radiol Bras. 2014;47:317-9.

22. Ceratti S, Pereira TR, Velludo SF, et al. Pulmonary tuberculosis in a patient with rheumatoid arthritis undergoing immunosuppressive treatment: case report. Radiol Bras. 2014;47:60-2.

23. Lachi T, Nakayama M. Radiological findings of pulmonary tuberculosis in indigenous patients in Dourados, MS, Brazil. Radiol Bras. 2015;48:275-81.

24. Guimarães MD. Pulmonary tuberculosis in Brazilian indians: a picture of this context depicted through radiography [Editorial]. Radiol Bras. 2015;48(5):v-vi.

25. Takahashi M, Tsukamoto H, Kawamura T, et al. Mycobacterium kansasii pulmonary infection: CT findings in 29 cases. Jpn J Radiol. 2012;30:398-406

26. Reich JM, Johnson RE. Mycobacterium avium complex pulmonary disease presenting as an isolated lingular or middle lobe pattern. The Lady Windermere syndrome. Chest. 1992;101:1605-9.

27. Reich JM. Pathogenesis of Lady Windermere syndrome. Scand J Infect Dis. 2012;44:1-2.

28. Christensen EE, Dietz GW, Ahn CH, et al. Pulmonary manifestations of Mycobacterium intracellularis. AJR Am J Roentgenol. 1979; 133:59-66.

29. Song JW, Koh WJ, Lee KS, et al. High-resolution CT findings of Mycobacterium avium-intracellulare complex pulmonary disease: correlation with pulmonary function test results. AJR Am J Roentgenol. 2008;191:1070. 